\title{
A Family of the Global Attractor for Higher Order Nonlinear Kirchhoff Equation
}

\author{
Guoguang Lin*, Yingguo Wang \\ Department of Mathematics, Yunnan University, Kunming, China \\ Email: ^gglin@ynu.edu.cn, 790612351@qq.com
}

How to cite this paper: Lin, G.G. and Wang, Y.G. (2021) A Family of the Global Attractor for Higher Order Nonlinear Kirchhoff Equation. Open Journal of Applied Sciences, 11, 750-765. https://doi.org/10.4236/ojapps.2021.116055

Received: May 18, 2021

Accepted: June 27, 2021

Published: June 30, 2021

Copyright $\odot 2021$ by author(s) and Scientific Research Publishing Inc. This work is licensed under the Creative Commons Attribution International License (CC BY 4.0).

http://creativecommons.org/licenses/by/4.0/

(c) (i) Open Access

\section{Abstract}

In this paper, we study the wellness and long time dynamic behavior of the solution of the initial boundary value problem for a class of higher order Kirchhoff equations $u_{t t}+M\left(\left\|D^{m} u\right\|_{p}^{p}\right)(-\Delta)^{2 m} u+\beta(-\Delta)^{2 m} u_{t}+g\left(u_{t}\right)=f(x)$ with strong damping terms. We will properly assume the stress term $M(s)$ and nonlinear term $g\left(u_{t}\right)$. First, we can prove the existence and uniqueness of the solution of the equation via a prior estimate and Galerkin's method, then the existence of the family of global attractor is obtained. At last, we can obtain that the Hausdorff dimension and Fractal dimension of the family of global attractors are finite.

\section{Keywords}

Kirchhoff-Type Equations, Prior Estimation, Galerkin's Method, The Family of Global Attractor, Hausdorff Dimension, Fractal Dimension

\section{Introduction}

This paper intends to study the initial-boundary value problem of higher-order Kirchhoff-type equation

$$
\begin{gathered}
u_{t t}+M\left(\left\|D^{m} u\right\|_{p}^{p}\right)(-\Delta)^{2 m} u+\beta(-\Delta)^{2 m} u_{t}+g\left(u_{t}\right)=f(x), \\
u(x, t)=0, \frac{\partial^{i} u}{\partial v^{i}}=0, i=1,2, \cdots, 2 m-1, x \in \partial \Omega, t>0, \\
u(x, 0)=u_{0}(x), u_{t}(x, 0)=u_{t}(x), x \in \Omega \subset R^{n} .
\end{gathered}
$$

where $m>1$, and $m \in N^{+}, \Omega \in R^{n}(n \geq 1)$ is a bounded domain, $\partial \Omega$ denotes the boundary of $\Omega, g\left(u_{t}\right)$ is a nonlinear source term, $\beta(-\Delta)^{2 m} u_{t}$ is a strongly dissipative term, $\beta>0, f(x)$ is an external force term. 
Kirchhoff-type equation model is one of the hot topics in mathematical physics equation research in recent years, which shows the importance of its position and influence. There have been many achievements in the study of the long-term behavior of the solution of Kirchhoff-type equation, for details, refer to references ([1] [2] [3] [4] [5]). Cheng Jianling and Yang Zhijian studied the asymptotic behavior of the solution of Kirchhoff-type equation in reference [6]:

$$
\begin{gathered}
u_{t t}-M\left(\|\Delta u\|^{2}\right) \Delta u-\Delta u_{t}+h\left(u_{t}\right)+g(u)=f(x),(x, t) \in \Omega \times R^{+}, \\
u(x, t)=0, \frac{\partial^{i} u}{\partial v^{i}}=0, i=1,2, \cdots, m-1, x \in \partial \Omega, t>0, \\
u(x, 0)=u_{0}, u_{t}=u_{1}(x), x \in \Omega .
\end{gathered}
$$

The existence of the global attractor of the corresponding operator semigroup $S(t)$ in phase space is proved.

Recently, Lin Guoguang et al. studied the existence of global attractors for higher order Kirchhoff type equations with nonlinear strong damping terms in reference [7]:

$$
\begin{gathered}
u_{t t}+(-\Delta)^{m} u_{t}+\phi\left(\left\|\nabla^{m} u\right\|^{2}\right)(-\Delta)^{m} u+h\left(u_{t}\right)=f(x), \\
u(x, t)=0, \frac{\partial^{i} u}{\partial v^{i}}=0, i=1,2, \cdots, m-1, x \in \partial \Omega, t>0, \\
u(x, 0)=u_{0}, u_{t}=u_{1}(x), x \in \Omega .
\end{gathered}
$$

They proved the existence and uniqueness of the solution of the equation by using prior estimation and Galerkin's method, and then obtained that the attractor exists in space $H^{2 m}(\Omega) \times H^{m}(\Omega)$.

Guoguang Lin and Changqing Zhu studied asymptotic state of solutions for a class of nonlinear higher order Kirchhoff type equations in reference [8]:

$$
\begin{gathered}
u_{t t}+M\left(\left\|D^{m} u\right\|^{2}\right)(-\Delta)^{m} u+\beta(-\Delta)^{m} u_{t}+g\left(x, u_{t}\right)=f(x), \\
u(x, t)=0, \frac{\partial^{i} u}{\partial v^{i}}=0, i=1,2, \cdots, m-1, x \in \partial \Omega, t>0, \\
u(x, 0)=u_{0}, u_{t}=u_{1}(x), x \in \Omega .
\end{gathered}
$$

For more results, please refer to references ([9]-[15]).

\section{Basic Assumptions}

For convenience, space and notations are defined as follows:

$$
H=L^{2}(\Omega), \quad D=\nabla, \quad H_{0}^{m}(\Omega)=H^{m}(\Omega) \cap H_{0}^{1}(\Omega),
$$

$H_{0}^{2 m+k}(\Omega)=H^{2 m+k}(\Omega) \cap H_{0}^{1}(\Omega), \quad E_{0}=H^{2 m}(\Omega) \times L^{2}(\Omega)$,

$E_{k}=H_{0}^{2 m+k}(\Omega) \times H_{0}^{k}(\Omega)$. Remember that $A_{k}$ is a family of global attractors from $E_{0}$ to $E_{k}, B_{0 k}$ is a bounded absorption set in $E_{k}$. In which $k=1,2, \cdots, 2 m, C_{i}(i=0,1,2, \cdots)$ is a constant; $(\cdot, \cdot),\|\cdot\|$ represent the inner product and norm on space H, namely $(u, v)=\int_{\Omega} u(x) v(x) \mathrm{d} x, \quad(u, u)=\|u\|^{2}$. 
Kirchhoff type stress term $M(s)$ satisfies the following conditions:

$\left(\mathrm{H}_{1}\right) \quad M(s) \in C^{2}([0,+\infty), R)$;

$\left(\mathrm{H}_{2}\right) \quad \varepsilon+1=\mu_{0} \leq M(s) \leq \mu_{1}$,

where $\mu_{0}, \mu_{1}$ are constant, $0<\varepsilon<\min \left\{\frac{2 \mu_{0} \lambda_{1}^{m}}{1+\beta \lambda_{1}^{m}}, \frac{\sqrt{4+2 \beta \lambda_{1}^{m}-2}}{2}, \frac{2}{\beta-1+\lambda_{1}^{-m}}\right\}$.

The nonlinear term $g\left(u_{t}\right)$ satisfies the following conditions:

$\left(\mathrm{H}_{3}\right)(g(s), s) \geq 0, \forall s \in H^{2 m}(\Omega) ;\|g(s)\| \leq(g(s), s)^{\partial}, \partial \in(0,1), \partial+\partial^{\prime}=1 ;$

$\left(\mathrm{H}_{4}\right)$ There is $\sigma \in(0,1),\|g(s)\| \leq C_{4}(R)\left(1+\varepsilon\left\|(-\Delta)^{k} s\right\|\right)^{1-\sigma}$,

$\forall s \in H^{4 m}(\Omega) \cap H_{0}^{2 m}(\Omega)$;

$\left(\mathrm{H}_{5}\right)\left\|g^{\prime}(s)\right\|_{\infty} \leq C_{3}$.

\section{The Existence of the Family of Global Attractor}

Lemma 3.1. set $M(s)$ satisfy assumption $\left(\mathrm{H}_{1}\right)$, $\left(u_{0}, u_{1}\right) \in E_{0}=H^{2 m}(\Omega) \times L^{2}(\Omega), \quad f(x) \in L^{2}(\Omega)$, and $u \in L^{\infty}\left(0,+\infty ; H^{2 m}(\Omega)\right)$, $v \in L^{\infty}\left(0,+\infty ; L^{2}(\Omega)\right) \cap L^{2}\left(0, T ; H^{2 m}(\Omega)\right)$, then the smooth solution of problems (1)-(3) $u(x, t)$ and $v(t)=u_{t}(x, t)+\varepsilon u(x, t)$ satisfy

$$
\|(u, v)\|_{E_{0}}^{2}=\left\|D^{2 m} u\right\|^{2}+\|v\|^{2} \leq w(0) \mathrm{e}^{-b t}+\frac{C_{1}}{b}\left(1-\mathrm{e}^{-b t}\right) .
$$

where $v=u_{t}+\varepsilon u, \quad w(0)=\left\|v_{0}\right\|^{2}+\varepsilon^{2}\left\|u_{0}\right\|^{2}+u\left\|\nabla^{2 m} u_{0}\right\|^{2}$, where constants $t_{0}$ and $R_{0}$, so that

$$
\|(u, v)\|_{E_{0}}^{2} \leq \frac{2 C_{1}}{b}=R_{0}\left(t>t_{0}\right) .
$$

Proof. It is proved that the inner product of $v=u_{t}+\varepsilon u$ and Equation (1.1) can be obtained

$$
\begin{aligned}
&\left(u_{t t}+M\left(\left\|D^{m} u\right\|_{p}^{p}\right)\right.\left.(-\Delta)^{2 m} u+\beta(-\Delta)^{2 m} u_{t}+g\left(u_{t}\right), v\right)=(f(x), v) . \\
&\left(u_{t t}, v\right)=\frac{1}{2} \frac{\mathrm{d}}{\mathrm{d} t}\|v\|^{2}+\frac{\varepsilon^{2}}{2} \frac{\mathrm{d}}{\mathrm{d} t}\|u\|^{2}-\varepsilon\|v\|^{2}+\varepsilon^{3}\|u\|^{2} . \\
&\left(M\left(\left\|\nabla^{m} u\right\|_{p}^{p}\right)(-\Delta)^{2 m} u, v\right)=\left(M\left(\left\|\nabla^{m} u\right\|_{p}^{p}\right) \nabla^{2 m} u, \nabla^{2 m}\left(u_{t}+\varepsilon u\right)\right) \\
&=\frac{M\left(\left\|\nabla^{m} u\right\|_{p}^{p}\right)}{2} \frac{\mathrm{d}}{\mathrm{d} t}\left\|\nabla^{2 m} u\right\|^{2}+\varepsilon M\left(\left\|\nabla^{m} u\right\|_{p}^{p}\right)\left\|\nabla^{2 m} u\right\|^{2} \\
& \geq \frac{\mu}{2} \frac{\mathrm{d}}{\mathrm{d} t}\left\|\nabla^{2 m} u\right\|^{2}+\varepsilon \mu_{0}\left\|\nabla^{2 m} u\right\|^{2} .
\end{aligned}
$$

By using the Poincare's inequality, we obtain

$$
\begin{aligned}
\left(\beta(-\Delta)^{2 m} u_{t}, v\right) & =\beta\left\|\nabla^{2 m} v\right\|^{2}-\left(\beta \varepsilon(-\Delta)^{2 m} u, v\right) \\
& \geq \frac{\beta}{2}\left\|\nabla^{2 m} v\right\|^{2}-\frac{\beta \varepsilon^{2}}{2}\left\|\nabla^{2 m} u\right\|^{2} \geq \frac{\beta \lambda_{1}^{2 m}}{2}\|v\|^{2}-\frac{\beta \varepsilon^{2}}{2}\left\|\nabla^{2 m} u\right\|^{2} \\
& \geq \frac{\beta}{4}\left\|D^{2 m} v\right\|^{2}+\frac{\beta \lambda_{1}^{2 m}}{4}\|v\|^{2}-\frac{\beta \varepsilon^{2}}{2}\left\|D^{2 m} u\right\|^{2} .
\end{aligned}
$$


By using the hypothesis $\left(\mathrm{H}_{3}\right)$ and Young's inequality, we obtain the following estimation

$$
\begin{aligned}
&\left(g\left(u_{t}\right), v\right)=\left(g\left(u_{t}\right), u_{t}+\varepsilon u\right)=\left(g\left(u_{t}\right), u_{t}\right)+\left(g\left(u_{t}\right), \varepsilon u\right) \\
& \geq\left(g\left(u_{t}\right), u_{t}\right)-\varepsilon\left\|g\left(u_{t}\right)\right\|-2 m \\
& \geq\left(g\left(u_{t}\right), u_{t}\right)-\varepsilon\left(g\left(u_{t}\right), u_{t}\right)^{\partial}\left\|D^{2 m} u\right\| \\
& \geq(1-\partial)\left(g\left(u_{t}\right), u_{t}\right)-\partial^{\prime} \varepsilon^{\frac{1}{\partial^{\prime}}}\left\|D^{2 m} u\right\|^{\frac{1}{\partial^{\prime}}} \\
& \geq(1-\partial)\left(g\left(u_{t}\right), u_{t}\right)-\frac{\varepsilon^{\frac{1}{\partial^{\prime}}}}{2}\left\|D^{2 m} u\right\|^{2}-\frac{\partial^{\prime} \varepsilon^{\frac{1}{\partial^{\prime}}}}{2 \partial^{\prime}-1} . \\
&(f(x), v) \leq\|f\|\|v\| \leq \frac{\varepsilon^{2}}{2}\|v\|^{2}+\frac{1}{2 \varepsilon^{2}}\|f\|^{2} .
\end{aligned}
$$

Substitute Inequality (3.2)-(3.6) into Equation (3.1), therefore,

$$
\begin{aligned}
& \frac{\mathrm{d}}{\mathrm{d} t}\left(\|v\|^{2}+\varepsilon^{2}\|u\|^{2}+\mu\left\|\nabla^{2 m} u\right\|^{2}\right)+\left(\frac{\beta \lambda_{1}^{2 m}}{2}-\varepsilon^{2}-2 \varepsilon\right)\|v\|^{2}+2 \varepsilon^{3}\|u\|^{2} \\
& +\left(2 \varepsilon \mu_{0}-\beta \varepsilon^{2}-\varepsilon^{\frac{1}{\partial^{\prime}}}\right)\left\|\nabla^{2 m} u\right\|^{2}+\frac{\beta}{2}\left\|D^{2 m} v\right\|^{2} \leq \frac{\|f\|^{2}}{\varepsilon^{2}}+\frac{2 \partial^{\prime} \varepsilon^{\frac{1}{\partial^{\prime}}}}{2 \partial^{\prime}-1} .
\end{aligned}
$$

let $b_{1}=\frac{\beta \lambda_{1}^{2 m}}{2}-\varepsilon^{2}-2 \varepsilon, b_{2}=2 \varepsilon \mu_{0}-\beta \varepsilon^{2}-\varepsilon^{\frac{1}{\hat{\sigma}^{\prime}}}$, and let $b=\min \left\{b_{1}, \frac{b_{2}}{\varepsilon}, \varepsilon^{2}\right\}$, $C_{1}=\frac{\|f\|^{2}}{\varepsilon^{2}}+\frac{2 \partial^{\prime} \varepsilon^{\frac{1}{\partial^{\prime}}}}{2 \partial^{\prime}-1}$, then

$$
\frac{\mathrm{d}}{\mathrm{d} t} w(t)+b w(t)+\frac{\beta}{2}\left\|D^{2 m} v\right\|^{2} \leq C_{1}
$$

where

$$
w(t)=\|v\|^{2}+\varepsilon^{2}\|u\|^{2}+\mu\left\|\nabla^{2 m} u\right\|^{2} .
$$

By using the Gronwall's inequality, we get

$$
w(t) \leq w(0) \mathrm{e}^{-b t}+\frac{C_{1}}{b}\left(1-\mathrm{e}^{-b t}\right) .
$$

where

$$
w(0)=\left\|v_{0}\right\|^{2}+\varepsilon^{2}\left\|u_{0}\right\|^{2}+\mu\left\|\nabla^{2 m} u_{0}\right\|^{2} .
$$

Then

$$
\|(u, v)\|_{H^{2 m} \times L^{2}}^{2}=\left\|D^{2 m} u\right\|^{2}+\|v\|^{2} \leq w(0) \mathrm{e}^{-b t}+\frac{C_{1}}{b}\left(1-\mathrm{e}^{-b t}\right),
$$

and

$$
\frac{\beta}{2} \int_{0}^{T}\left\|\Delta^{m} v\right\|^{2} \mathrm{~d} t \leq C_{1} T
$$

There are constants $t_{0}(\Omega)$ and $R_{0}$, we, get 


$$
\|(u, v)\|_{E_{0}}^{2} \leq \frac{2 C_{1}}{b}=R_{0}\left(t>t_{0}\right) .
$$

Lemma 3.1 is proved.

Lemma 3.2. If lemma 3.1 holds, and the condition is $\left(\mathrm{H}_{4}\right)$, set $\left(u_{0}, v_{0}\right) \in H^{2 m+k}(\Omega) \times H^{k}(\Omega), \quad k=1,2, \cdots, m . f \in H^{k}(\Omega)$, then the smooth solution of Problems (1.1)-(1.3) $u(x, t)$ and $v(t)=u_{t}(x, t)+\varepsilon u(x, t)$ satisfy

$$
\|(u, v)\|_{H^{2 m+k} \times H^{k}}^{2}=\left\|D^{2 m+k} u(t)\right\|^{2}+\left\|D^{k} v(t)\right\|^{2} \leq M(0) \mathrm{e}^{-\alpha t}+\frac{C_{2}}{\alpha}\left(1-\mathrm{e}^{-\alpha t}\right),
$$

where constants $t_{1}(\Omega)$ and $R_{1}$, then

$$
\|(u, v)\|_{H^{2 m+k} \times H^{k}}^{2} \leq \frac{2 C_{2}}{\alpha}=R_{1}^{2},\left(t>t_{1}(\Omega)\right) .
$$

Proof. Set $(-\Delta)^{k} v=(-\Delta)^{k} u_{t}+\varepsilon(-\Delta)^{k} u$. It is obtained by inner product of $(-\Delta)^{k} v$ and Formula (1.1).

$$
\begin{gathered}
\left(u_{t t}+M\left(\left\|D^{m} u\right\|_{p}^{p}\right)(-\Delta)^{2 m} u+\beta(-\Delta)^{2 m} u_{t}+g\left(u_{t}\right),(-\Delta)^{k} v\right)=\left(f(x),(-\Delta)^{k} v\right) . \\
\left(u_{t t},(-\Delta)^{k} v\right)=\left(v_{t}-\varepsilon u_{t},(-\Delta)^{k} v\right)=\frac{1}{2} \frac{\mathrm{d}}{\mathrm{d} t}\left\|D^{k} v\right\|^{2}-\varepsilon\left\|D^{k} v\right\|^{2}+\varepsilon^{2}\left(u,(-\Delta)^{k} v\right) \\
=\frac{1}{2} \frac{\mathrm{d}}{\mathrm{d} t}\left\|D^{k} v\right\|^{2}-\varepsilon\left\|D^{k} v\right\|^{2}+\frac{\varepsilon^{2}}{2} \frac{\mathrm{d}}{\mathrm{d} t}\left\|D^{k} u\right\|^{2}+\varepsilon^{3}\left\|D^{k} u\right\|^{2} . \\
\left(\beta(-\Delta)^{2 m} u_{t},(-\Delta)^{k} v\right)=\left(\beta(-\Delta)^{2 m}(v-\varepsilon u),(-\Delta)^{k} v\right) \\
=\left(\beta(-\Delta)^{2 m} v,(-\Delta)^{k} v\right)-\varepsilon \beta\left((-\Delta)^{2 m} u,(-\Delta)^{k} v\right) \\
\geq \beta\left\|D^{2 m+k} v\right\|^{2}-\frac{\beta \varepsilon^{2}}{2}\left\|D^{2 m+k} u\right\|^{2}-\frac{\beta \lambda_{1}^{2 m}}{2}\left\|D^{k} v\right\|^{2} \\
\geq \frac{\beta}{3}\left\|D^{2 m+k} v\right\|^{2}-\frac{\varepsilon^{2} \beta}{2}\left\|D^{2 m+k} u\right\|^{2}+\frac{\lambda_{1}^{2 m} \beta}{6}\left\|D^{k} v\right\|^{2} . \\
\left(M\left(\left\|D^{m} u\right\|_{p}^{p}\right)(-\Delta)^{2 m} u,(-\Delta)^{k} v\right) \geq \frac{\delta}{2} \frac{\mathrm{d}}{\mathrm{d} t}\left\|D^{2 m+k} u\right\|^{2}+\varepsilon \delta_{0}\left\|D^{2 m+k} u\right\|^{2} .
\end{gathered}
$$

From hypothesis $\left(\mathrm{H}_{4}\right)$, we get

$$
\begin{aligned}
& \left(g\left(u_{t}\right),(-\Delta)^{k} v\right) \\
& \leq\left\|g\left(u_{t}\right)\right\|\left\|D^{2 k} v\right\| \\
& \leq C_{4}\left\|D^{2 k} v\right\|\left(1+\varepsilon\left\|(-\Delta)^{k} u_{t}\right\|\right)^{1-\sigma} \\
& \leq C_{4}\left\|D^{2 k} v\right\|\left(1+\varepsilon\left\|D^{2 k} v-\varepsilon D^{2 k} u\right\|\right)^{1-\sigma} \\
& \leq C_{4}\left\|D^{2 k} v\right\|\left(1+\varepsilon\left\|D^{2 k} v\right\|+\varepsilon^{2}\left\|D^{2 k} u\right\|\right)^{1-\sigma} \\
& \leq C_{4}\left\|D^{2 k} v\right\|\left(1+\varepsilon\left\|D^{2 k} v\right\|+\varepsilon^{2}\left\|D^{2 k} u\right\|\right) \\
& \leq C_{4}\left\|D^{2 k} v\right\|+C_{4} \varepsilon\left\|D^{2 k} v\right\|^{2}+C_{4} \varepsilon^{2}\left\|D^{2 k} v\right\|\left\|D^{2 k} u\right\|
\end{aligned}
$$




$$
\begin{aligned}
& \leq C_{5}+C_{4}\left(\varepsilon+\varepsilon^{2}\right)\left\|D^{2 k} v\right\|^{2}+\frac{C_{4}}{2} \varepsilon^{2}\left\|D^{2 k} u\right\|^{2} \\
& \leq C_{5}+C_{4}\left(\varepsilon+\varepsilon^{2}\right) \lambda_{1}^{2 m-k}\left\|D^{2 m+k} v\right\|^{2}+C_{4} \varepsilon^{2} \lambda_{1}^{2 m-k}\left\|D^{2 m+k} u\right\|^{2} .
\end{aligned}
$$

Then

$$
\begin{gathered}
\left(g\left(u_{t}\right),(-\Delta)^{k} v\right) \geq-C_{5}-C_{4}\left(\varepsilon+\varepsilon^{2}\right) \lambda_{1}^{2 m-k}\left\|D^{2 m+k} v\right\|^{2}-C_{4} \varepsilon^{2} \lambda_{1}^{2 m-k}\left\|D^{2 m+k} u\right\|^{2} . \\
\left(f(x),(-\Delta)^{k} v\right)=\left(\nabla^{k} f(x), \nabla^{k} v\right) \leq \frac{\left\|\nabla^{k} f\right\|^{2}}{2 \varepsilon^{2}}+\frac{\varepsilon^{2}}{2}\left\|\nabla^{k} v\right\|^{2} .
\end{gathered}
$$

Substitute Inequality (3.16)-(3.20) into Equation (3.15), therefore,

$$
\begin{aligned}
& \frac{\mathrm{d}}{\mathrm{d} t}\left(\left\|D^{k} v\right\|^{2}+\varepsilon^{2}\left\|D^{k} u\right\|^{2}+\delta\left\|D^{2 m+k} u\right\|^{2}\right)+\left(\frac{\beta \lambda_{1}^{2 m}}{3}-2 \varepsilon-\varepsilon^{2}\right)\left\|D^{k} v\right\|^{2} \\
& +2 \varepsilon^{3}\left\|D^{k} u\right\|^{2}+\left(2 \varepsilon \delta_{0}-\beta \varepsilon^{2}-C_{4} \varepsilon^{2} \lambda_{1}^{2 m-k}\right)\left\|D^{2 m+k} u\right\|^{2} \\
& +\left[\frac{\beta}{3}-C_{4}\left(\varepsilon+\varepsilon^{2}\right) \lambda_{1}^{2 m-k}\right]\left\|D^{2 m+k} v\right\|^{2}+\frac{\beta}{3}\left\|D^{2 m+k} v\right\|^{2} \leq \frac{\left\|\nabla^{k} f\right\|^{2}}{\varepsilon^{2}}+C_{5} .
\end{aligned}
$$

Let $\alpha_{1}=\frac{\beta \lambda_{1}^{2 m}}{3}-2 \varepsilon-\varepsilon^{2} \geq 0$,

$$
\begin{gathered}
\alpha_{2}=2 \varepsilon \delta_{0}-\beta \varepsilon^{2}-C_{4} \varepsilon^{2} \lambda_{1}^{2 m-k} \geq 0, \\
\frac{\beta}{3}-C_{4}\left(\varepsilon+\varepsilon^{2}\right) \lambda_{1}^{2 m-k} \geq 0, \text { and let } \alpha=\min \left\{\alpha_{1} \frac{\alpha_{2}}{\delta}, \varepsilon^{3}\right\}, C_{2}=\frac{\left\|\nabla^{k} f\right\|^{2}}{\varepsilon^{2}}+C_{5},
\end{gathered}
$$

then

$$
\frac{\mathrm{d}}{\mathrm{d} t} M(t)+\alpha M(t)+\frac{2 \beta}{3}\left\|D^{2 m+k} v\right\|^{2} \leq C_{2} .
$$

where

$$
M(t)=\left\|D^{k} v\right\|^{2}+\varepsilon^{2}\left\|D^{k} u\right\|^{2}+\delta \frac{\mathrm{d}}{\mathrm{d} t}\left\|D^{2 m+k} u\right\|^{2} .
$$

By using the Gronwall's inequality, we get

$$
M(t) \leq M(0) \mathrm{e}^{-\alpha t}+\frac{C_{2}}{\alpha}\left(1-\mathrm{e}^{-\alpha t}\right),
$$

where

$$
M(0)=\left\|D^{k} v_{0}\right\|^{2}+\varepsilon^{2}\left\|D^{k} u_{0}\right\|^{2}+\delta \frac{\mathrm{d}}{\mathrm{d} t}\left\|D^{2 m+k} u_{0}\right\|^{2} .
$$

Then

$$
\|(u, v)\|_{H^{2 m+k} \times H^{k}}^{2}=\left\|D^{2 m+k} u(t)\right\|^{2}+\left\|D^{k} v(t)\right\|^{2} \leq M(0) \mathrm{e}^{-\alpha t}+\frac{C_{2}}{\alpha}\left(1-\mathrm{e}^{-\alpha t}\right),
$$

and

$$
\frac{\beta}{3} \int_{0}^{T}\left\|D^{2 m+k} v\right\|^{2} \mathrm{~d} t \leq C_{2} T .
$$


There are constants $t_{1}(\Omega)$ and $R_{1}$, then

$$
\|(u, v)\|_{H^{2 m+k} \times H^{k}}^{2} \leq \frac{2 C_{2}}{\alpha}=R_{1}^{2} .
$$

Lemma 3.2 is proved.

Theorem 3.1. Assume that the nonlinear function $M(s)$ satisfies $\left(\mathrm{H}_{1}\right),\left(\mathrm{H}_{2}\right)$, $\left(u_{0}, u_{i}\right) \in E_{0}, \quad f(x) \in L^{2}(\Omega)$, then the Problems (1.1)-(1.3) have a unique global smooth solution $(u, v) \subset L^{\infty}\left([0,+\infty), E_{0}\right)$, and $v \in L^{2}\left(0, T ; H_{0}^{2 m}(\Omega)\right)$.

Proof: The proof of existence is divided into the following three steps by Galekin's method:

Step 1: Approximate solution

Suppose the eigenvector $w_{j}$ of $(-\Delta)^{2 m} \omega_{j}=\lambda_{j}^{2 m} \omega_{j}$ generates an orthonormal basis for $H^{2 m}$, where $\lambda_{j}$ is the eigenvalue of $-\Delta$ with homogeneous Dirichlet boundary on $\Omega$, define k order approximation $u_{k}(t)$ :

$$
\begin{aligned}
& u_{k}(t) \in \operatorname{span}\left[\omega_{1}, \omega_{2}, \cdots, \omega_{k}\right], u_{k}(t)=\sum_{j=1}^{k} g_{j k}(t) \omega_{j} . \\
& \left(u_{k}^{\prime \prime}(t)+M\left(\left\|D^{m} u_{k}(t)\right\|_{P}^{P}\right)(-\Delta)^{2 m} u_{k}(t)+\beta(-\Delta)^{2 m} u_{k}^{\prime}(t)+g\left(u_{k}\right), \omega_{j}\right) \\
& =\left(f(x), \omega_{j}\right) .
\end{aligned}
$$

where $1<j<k$,

$$
u_{k}(0)=v_{0 k}, u_{k}^{\prime}(0)=u_{1 k}, u_{0 k}, u_{1 k} \in \operatorname{span}\left[\omega_{1}, \omega_{2}, \cdots, \omega_{k}\right] .
$$

with in $\mathrm{H}$,

$$
u_{0 k} \rightarrow u_{0}, u_{1 k} \rightarrow u_{1} .
$$

This system of ordinary differential equations about $g_{j k}(t)$ can determine $u_{k}(t)$ in the interval $\left[0, t_{k}\right]$; need to prove $t_{k}=T$.

Step 2: Prior estimation

According to the conclusion and proof method of lemma 3.1, $\left(u_{k}(t), u_{k}^{\prime}(t)\right)$ is uniformly bounded on $E_{0}$, then

$$
\begin{gathered}
\left\|u_{k}(t)\right\| \leq R \\
\left\|D^{2 m} u_{k}(t)\right\| \leq R \\
\left\|u_{k}^{\prime}(t)\right\| \leq R
\end{gathered}
$$

thus it can be seen $t_{k}=T$, Inequality (3.32)-(3.33) shows $u_{k}(t)$ is bounded in $L^{\infty}\left(0, T ; H^{2 m}(\Omega)\right)$, and $u_{k}^{\prime}(t)$ is bounded in $L^{\infty}\left(0, T ; L^{2}(\Omega)\right)$.

And it's actually available $u_{k}(t)$ in $L^{\infty}\left(0,+\infty ; H^{2 m}(\Omega)\right)$ and $u_{k}^{\prime}(t)$ in $L^{\infty}\left(0,+\infty ; L^{2}(\Omega)\right)$.

Step 3: Limit process

According to Danford-Pttes theorem, Space $L^{\infty}\left(0, T ; H^{2 m}(\Omega)\right)$ conjugate to space $L^{\prime}\left(0, T ; H^{-2 m}(\Omega)\right)$; Space $L^{\infty}\left(0, T ; L^{2}(\Omega)\right)$ conjugate to space $L^{\prime}\left(0, T ; L^{2}(\Omega)\right)$, select the subsequence $u_{h}(t)$ from the sequence $u_{k}(t)$, such that 
$u_{h}(t) \rightarrow u$ weakly * converges in $L^{\infty}\left(0, T ; H^{2 m}(\Omega)\right)$,

$u_{h}^{\prime}(t) \rightarrow u^{\prime}$ weakly * converges in $L^{\infty}\left(0, T ; L^{2}(\Omega)\right)$,

$u_{h}^{\prime}(t) \rightarrow u^{\prime}$ weakly converges in $L^{2}\left(0, T ; H^{2 m}(\Omega)\right)$.

According to Pellich-Kohdarachov theorem, $H^{2 m}(\Omega)$ is compact embedded in $L^{P}(\Omega)$ and $L^{2}(\Omega), u_{k}(t) \rightarrow u$ is strong convergence almost everywhere in $L^{P}(0, T)$ and $L^{2}(0, T)$.

$$
\begin{aligned}
& M\left(\left\|u_{k}(t)\right\|_{p}^{p}\right) \rightarrow M\left(\|u(t)\|_{p}^{p}\right) \text { converges in } R^{+} . \\
& M\left(\left\|u_{k}(t)\right\|_{p}^{p}\right)\left((-\Delta)^{2 m} u_{k}(t), D^{2 m} w_{j}(t)\right) \rightarrow M\left(\|u(t)\|_{p}^{p}\right)\left((-\Delta)^{2 m} u_{k}(t), D^{2 m} w_{j}(t)\right)
\end{aligned}
$$
is weak ${ }^{*}$ convergence in $L^{\infty}(0, T)$.

$\beta\left(\nabla^{2 m} u_{k}^{\prime}(t), \nabla^{2 m} w_{j}(t)\right) \rightarrow \beta\left(\nabla^{2 m} u^{\prime}(t), \nabla^{2 m} w_{j}\right)$ is weak * convergence in $L^{\infty}(0, T)$.

$\left(u_{h}(t), \omega_{j}\right)=\frac{\mathrm{d}}{\mathrm{d} x}\left(u_{k}^{\prime}(t), \omega_{j}\right) \rightarrow\left(u^{\prime \prime}(t), \omega_{j}\right)$ converges in $D^{\prime}(0, T)$.

$\left(g\left(u_{k}^{\prime}(t), \omega_{j}\right)\right) \rightarrow\left(g\left(u^{\prime}(t), \omega_{j}\right)\right)$ is weak ${ }^{*}$ convergence in $L^{\infty}(0, T)$.

From the Formula (3.30), we get

$$
\begin{aligned}
& \left(u^{\prime \prime}(t), \omega_{j}\right)+\left(M\left(\left\|D^{m} u\right\|_{p}^{p}\right)\right)\left(\nabla^{2 m} u, \nabla^{2 m} \omega_{j}\right)+\beta\left(\nabla^{2 m} u^{\prime}(t), \nabla^{2 m} w_{j}\right) \\
& +\left(g\left(u_{t}\right), \omega_{j}\right)=\left(f(x), \omega_{j}\right)
\end{aligned}
$$

For $\forall j$, according to the density of $\omega_{1}, \omega_{2}, \cdots, \omega_{k}, \cdots$.

$$
\begin{aligned}
& \left(u^{\prime \prime}, \varphi\right)+\left(M\left(\left\|D^{m} u\right\|_{p}^{p}\right)\right)\left(\nabla^{2 m} u, \nabla^{2 m} \varphi\right)+\beta\left(\nabla^{2 m} u^{\prime}(t), \nabla^{2 m} \varphi\right)+\left(g\left(u_{t}\right), \varphi\right) \\
& =(f(x), \varphi)
\end{aligned}
$$

$\forall \varphi \in H^{2 m}(\Omega)$ and $u_{k}(0) \rightarrow u(0)$ weakly converges in $L^{2}(\Omega)$, and in $H^{2 m}(\Omega)$.

$$
\begin{gathered}
u_{k}(0) \rightarrow u(0), \\
\left.\left(u_{k}^{\prime}(0), \omega_{j}\right) \rightarrow\left(u^{\prime}, \omega_{j}\right)\right|_{t=0}=\left(u^{\prime}(0), \omega_{j}\right), \\
\left(u_{k}^{\prime}(0), \omega_{j}\right) \rightarrow\left(u_{1}, \omega_{j}\right),
\end{gathered}
$$

then $\left(u^{\prime}(0), \omega_{j}\right) \rightarrow\left(u_{1}, \omega_{j}\right)$ is satisfied for all $\mathrm{j}$, so that existence can be proved.

Then prove the uniqueness of the solution.

Set $u^{*}, v^{*}$ be two solutions of the Problem (1.1)-(1.3), let $\omega=u^{*}-v^{*}$, then

$$
\begin{aligned}
& \omega_{t t}+\beta(-\Delta)^{2 m} \omega_{t}+M\left(\left\|D^{m} u^{*}(t)\right\|_{p}^{p}\right)(-\Delta)^{2 m} u^{*}-M\left(\left\|D^{m} v^{*}(t)\right\|_{p}^{p}\right)(-\Delta)^{2 m} v^{*} \\
& +g\left(u_{t}^{*}\right)-g\left(v_{t}^{*}\right)=0,
\end{aligned}
$$

Take the inner product with $\omega_{t}$ and (3.34).

$$
\begin{aligned}
& \left(\omega_{t t}+\beta(-\Delta)^{2 m} \omega_{t}+M\left(\left\|D^{m} u^{*}(t)\right\|_{p}^{p}\right)(-\Delta)^{2 m} u^{*}, \omega_{t}\right) \\
& -\left(M\left(\left\|D^{m} v^{*}(t)\right\|_{p}^{p}\right)(-\Delta)^{2 m} v^{*}+g\left(u_{t}^{*}\right)-g\left(v_{t}^{*}\right), \omega_{t}\right)=0 .
\end{aligned}
$$




$$
\begin{gathered}
\left(\omega_{t t}, \omega_{t}\right)=\frac{1}{2} \frac{\mathrm{d}}{\mathrm{d} t}\left\|\omega_{t}\right\|^{2} . \\
\beta\left((-\Delta)^{2 m} \omega_{t}, \omega_{t}\right) \geq \frac{\beta}{2}\left\|D^{2 m} \omega_{t}\right\|^{2} .
\end{gathered}
$$

By using hypothesis $\left(\mathrm{H}_{5}\right)$, there are $\left\|g^{\prime}(s)\right\|_{\infty} \leq C_{3}$, then

$$
\left|\left(\left(g\left(u_{t}^{*}\right)-g\left(v_{t}^{*}\right)\right), w_{t}\right)\right|=\left|\left(g^{\prime}(s) w_{t}, w_{t}\right)\right| \leq C_{3}\left\|w_{t}\right\|^{2} .
$$

By using hypothesis $\left(\mathrm{H}_{1}\right)$, Lemma 3.1 and Differential Mean Value Theorem

$$
\begin{aligned}
& \left(M\left(\left\|D^{m} u^{*}\right\|_{p}^{p}\right)(-\Delta)^{2 m} u^{*}-M\left(\left\|D^{m} v^{*}(t)\right\|_{p}^{p}\right)(-\Delta)^{2 m} v^{*}, w_{t}\right) \\
& =\frac{1}{2}\left(M\left(\left\|D^{m} u^{*}\right\|_{p}^{p}\right) \frac{\mathrm{d}}{\mathrm{d} t}\left\|D^{2 m} w\right\|^{2}+M\left(\left\|D^{m} u^{*}(t)\right\|_{p}^{p}-M\left(\left\|D^{m} v^{*}\right\|_{p}^{p}\right)\right)(-\Delta)^{2 m} v^{*}, w_{t}\right) \\
& \geq \frac{1}{2} \mu \frac{\mathrm{d}}{\mathrm{d} t}\left\|D^{2 m} w\right\|^{2}-\left\|M^{\prime}(\xi)\right\|_{\infty} C_{6}\left(\left\|D^{m} w\right\|_{p-1}^{p-1}\right) \cdot\left\|D^{m} w\right\| \cdot\left\|D^{2 m} v^{*}\right\|\left\|D^{2 m} w_{t}\right\| \\
& \geq \frac{1}{2} \mu \frac{\mathrm{d}}{\mathrm{d} t}\left\|D^{2 m} w\right\|^{2}-C_{7}\left\|D^{m} w\right\|\left\|D^{2 m} w_{t}\right\| \\
& \geq \frac{1}{2} \mu \frac{\mathrm{d}}{\mathrm{d} t}\left\|D^{2 m} w\right\|^{2}-\frac{\beta}{2}\left\|D^{2 m} w_{t}\right\|^{2}-\frac{C_{7}^{2}}{2 \beta}\left\|D^{m} w\right\|^{2} .
\end{aligned}
$$

To sum up, we obtain

$$
\frac{\mathrm{d}}{\mathrm{d} t}\left(\left\|w_{t}\right\|^{2}+\mu\left\|D^{2 m} w\right\|^{2}\right) \leq \frac{C_{7}^{2}}{\beta}\left\|D^{m} w\right\|^{2} \leq \frac{C_{7}^{2}}{\mu \beta \lambda_{1}^{m}}\left(\left\|w_{t}\right\|^{2}+\mu\left\|D^{2 m} w\right\|^{2}\right) .
$$

From Gronwall's inequality, we get

$$
\left\|w_{t}\right\|^{2}+\mu\left\|D^{2 m} w(t)\right\|^{2} \leq \mathrm{e}^{\frac{C_{7}^{2}}{\mu \beta \lambda_{1}^{m}}}\left(\|w(0)\|^{2}+\mu\left\|D^{2 m} w_{1}(0)\right\|^{2}\right)=0 .
$$

Therefore $u^{*}=v^{*}$, the uniqueness is proved.

Theorem 3.2. [9] Let $E$ be a Banach space, $S(t): E \rightarrow E$ Semigroups satisfy the following conditions

1) Semigroup $S(t)$ is uniformly bounded in $E$, then $\forall r>0$, there is constant $C(r)$, so that when $\|u\|_{E} \leq r$, there is $\|S(t) u\|_{E} \leq C(r),(\forall t \in[0,+\infty))$;

2) There is a bounded absorption set $B_{0}$ in $E$;

3) $S(t)$ is a fully continuous operator. That is, semigroups $S(t)$ have compact global attractors $A_{0}$.

The Banach space $E$ in theorem 3.2 is changed into Hilbert space $E_{k}$, there are the following existence theorems of global attractor's families.

Theorem 3.3. Under the hypothesis of lemma 3.1 and lemma 3.2, Then there is a family of global attractor $A_{k}(k=1,2, \cdots, 2 m)$ for Problems (1.1)-(1.3). That is, there is a compact set $A_{k} \subset E_{k} \subset E_{0}$, make:

1) $S(t) A_{k}=A_{k}, \forall t>0$;

2) $\lim _{t \rightarrow \infty} \operatorname{dist}\left(S(t) B_{k}, A_{k}\right)=0 \quad\left(\forall B_{k} \subset E_{k}\right.$ is a bounded set), among $\lim _{t \rightarrow \infty} \operatorname{dist}\left(S(t) B_{k}, A_{k}\right)=\sup _{x \in B_{k}} \inf _{y \in A_{k}}\|S(t) x-y\|_{E_{k}}, S(t)$ is the solution semigroup generated by Problems (1.1)-(1.3). 
Proof: It is necessary to verify the conditions (1), (2) and (3) of Theorem 3.2. Solution Semigroup $S(t): E_{k} \rightarrow E_{k}$ generated by Theorem 3.1 and Lemma 3.2 to know Problems (1.1)-(1.3).

1) Knowing the arbitrary bounded set $B_{k} \subset E_{k}$ by Lemma 3.2, have $\|(u, v)\|_{H^{2 m+k} \times H^{k}}^{2} \leq R_{1}^{2}$ and

$$
\left\|S(t)\left(u_{0}, v_{0}\right)\right\|_{E_{k}}^{2}=\|u\|_{H_{0}^{2 m+k}(\Omega)}^{2}+\|v\|_{H_{0}^{k}(\Omega)}^{2} \leq\left\|u_{0}\right\|_{H_{0}^{2 m+k}(\Omega)}^{2}+\left\|v_{0}\right\|_{H_{0}^{k}(\Omega)}^{2} \leq R_{k}^{2} .
$$

where $t \geq 0,\left(u_{0}, v_{0}\right) \in B_{k}$, this shows that $\{S(t)\}(t \geq 0)$ is uniformly bounded with in $E_{k}$.

2) Further, about $\forall\left(u_{0}, v_{0}\right) \in E_{k}$, there is

$$
\left\|S(t)\left(u_{0}, v_{0}\right)\right\|_{E_{k}}^{2}=\|u\|_{H_{0}^{2 m+k}(\Omega)}^{2}+\|v\|_{H_{0}^{k}(\Omega)}^{2} \leq R_{1}^{2}+R_{0}^{2} \leq R_{k}^{2} .
$$

Then $B_{0 k}$ is a bounded absorbing set of semigroup $S(t)$.

3) According to Rellich-Kondrachov compact embedding theorem $E_{k} \subset E_{0}$, then the bounded set in $E_{k}$ is the compact set in $E_{0}$, therefore, the solution semigroup $S(t)$ is a fully continuous operator. Therefore, the family of global attractor $A_{k}$ of the solution semigroup $S(t)$ can be obtained, where

$$
A_{k}=W\left(B_{0 k}\right)=\bigcap_{0 \leq \tau \leq t} \overline{\bigcup S(t) B_{0 k}} .
$$

The theorem is completed.

\section{Dimension Estimation of the Family of Global Attractor}

First consider the linearization of Problems (1.1)-(1.3)

$$
\begin{gathered}
U_{t t}+M\left\|D_{u}^{m}\right\|_{p}^{p}(-\Delta)^{2 m} U+M\left\|D_{u}^{m}\right\|_{p}^{p}\left(\left\|D_{u}^{m}\right\|_{p}^{p}\right)^{\prime}(-\Delta)^{2 m} U \\
+\beta(-\Delta)^{2 m} U_{t}+g^{\prime}\left(u_{t}\right) U_{t}=0 . \\
\left.U(x, t)\right|_{\partial \Omega}=\left.(-\Delta)^{k} U(x, t)\right|_{\partial \Omega}=0, k=1, \cdots, 2 m-1, t>0, \\
U(x, 0)=\xi_{1}, U_{t}(x, 0)=\xi_{2} .
\end{gathered}
$$

where $\left(\xi_{1}, \xi_{2}\right) \in E_{k},\left(u, u_{t}\right)=S(t)\left(u_{0}, u_{1}\right)$ is the solution of Problems (1.1)-(1.3), known $\left(u_{0}, u_{1}\right) \in A_{k}, S(t): E \rightarrow E$, can prove $\forall\left(\xi_{1}, \xi_{2}\right) \in E_{k}$, the linearization Problems (4.1)-(4.3) have unique solutions $\left(U(t), U_{t}(t)\right) \in L^{\infty}\left((0,+\infty) ; E_{k}\right)$.

Theorem 4.1. $\forall t>0, R>0$, the mapping $S(t): E_{k} \rightarrow E_{k}$ is Frechet differentiable on $E_{k}$, and differentiate the linear operator of $G(t):\left(\xi_{1}, \xi_{2}\right)^{\mathrm{T}} \rightarrow\left(U(t), U_{t}(t)\right)^{\mathrm{T}}$, where $U(t), U_{t}(t)$ is the solution of problems (4.1)-(4.3).

Proof: Set $\phi_{0}=\left(u_{0}, u_{1}\right)^{\mathrm{T}} \in E_{k}, \overline{\phi_{0}}=\left(u_{0}+\xi_{1}, u_{1}+\xi_{2}\right)^{\mathrm{T}} \in E_{k}$, then $\left\|\phi_{0}\right\|_{E_{k}} \leq R,\left\|\bar{\phi}_{0}\right\|_{E_{k}} \leq R$, from this we can get the Lipchitz property of $S(t)$ on the bounded set of $E_{k}$, that is,

$$
\left\|S(t) \overline{\phi_{0}}-S(t) \phi_{0}\right\|_{E_{k}}^{2} \leq \mathrm{e}^{c t}\left\|\left(\xi_{1}, \xi_{2}\right)\right\|_{E_{k}}^{2} .
$$


Set $\sigma=\tilde{u}-u-U$, then

$$
\begin{gathered}
\sigma_{t t}+M\left(\left\|D^{m} u\right\|_{p}^{p}\right)(-\Delta)^{2 m} \sigma+\beta(-\Delta)^{2 m} \sigma_{t}=h . \\
\sigma(0)=\xi_{1}, \sigma_{t}(0)=\xi_{2},
\end{gathered}
$$

let $s=\left\|\nabla^{m} u\right\|_{p}^{p}, \tilde{s}=\left\|\nabla^{m} \tilde{u}\right\|_{p}^{p}$,

then

$$
\begin{aligned}
h= & (M(s)-M(\tilde{s}))(-\Delta)^{2 m} \tilde{u}+p M^{\prime}(s)\left\|\nabla^{m} u\right\|^{p-2}\left\|\nabla^{m} u\right\|\left\|D^{m} U\right\|(-\Delta)^{2 m} u \\
& +g\left(u_{t}\right)-g\left(\overline{u_{t}}\right)+g^{\prime}\left(u_{t}\right) U_{t} .
\end{aligned}
$$

Take the inner product of $(-\Delta)^{k} \sigma_{t}$ and both sides of Formula (4.5)

$$
\frac{1}{2} \frac{\mathrm{d}}{\mathrm{d} t}\left\|D^{k} \sigma_{t}\right\|^{2}+\frac{1}{2} M(s) \frac{\mathrm{d}}{\mathrm{d} t}\left\|D^{2 m+k} \sigma\right\|^{2}+\beta\left\|D^{2 m+k} \sigma_{t}\right\|^{2}=\left(h,(-\Delta)^{k} \sigma_{t}\right) .
$$

Let $\bar{u}=u-\tilde{u}$, according to lemma 3.2, differential mean value theorem and poincare's inequality

$$
\begin{aligned}
& \left((M(s)-M(\tilde{s}))(-\Delta)^{2 m} \tilde{u}+p M^{\prime}(s)\left\|\nabla^{m} u\right\|^{p-2}\left\|\nabla^{m} u\right\|\left\|D^{m} U\right\|(-\Delta)^{2 m} u,(-\Delta)^{k} \sigma_{t}\right) \\
& =\left(M^{\prime}(a s+(1-a) \tilde{s})(s-\tilde{s})(-\Delta)^{2 m} \tilde{u}+p M^{\prime}(s)\left\|\nabla^{m} u\right\|^{p-2}\left\|\nabla^{m} u\right\|\left\|D^{m} U\right\|(-\Delta)^{2 m} u,(-\Delta)^{k} \sigma_{t}\right) \\
& \geq\left(M^{\prime}(a s+(1-a) \tilde{s})\left(\nabla^{m} \bar{u},\left\|\nabla^{m} u\right\|^{p-1}+\left\|\nabla^{m} \tilde{u}\right\|^{p-1}\right)(-\Delta)^{2 m} \tilde{u},(-\Delta)^{k} \sigma_{t}\right) \\
& +\left(p M^{\prime}(s)\left\|\nabla^{m} u\right\|^{p-2}\left\|\nabla^{m} u\right\|\left\|D^{m} U\right\|(-\Delta)^{2 m} u,(-\Delta)^{k} \sigma_{t}\right) \\
& =I_{1}+I_{2}+I_{3} .
\end{aligned}
$$

$$
\begin{aligned}
I_{1}= & \left(M^{\prime}(a s+(1-a) \tilde{s})\left(\nabla^{m} \bar{u},\left\|\nabla^{m} u\right\|^{p-1}+\left\|\nabla^{m} \tilde{u}\right\|^{p-1}\right)(-\Delta)^{2 m} \tilde{u},(-\Delta)^{k} \sigma_{t}\right) \\
& -\left(M^{\prime}(s)\left(\nabla^{m} \bar{u},\left\|\nabla^{m} u\right\|^{p-1}+\left\|\nabla^{m} \tilde{u}\right\|^{p-1}\right)(-\Delta)^{2 m} \tilde{u},(-\Delta)^{k} \sigma_{t}\right) \\
= & \left(M^{\prime \prime}(\xi)(a s+(1-a) \tilde{s}-s)\right)\left(\nabla^{m} \bar{u},\left\|\nabla^{m} u\right\|^{p-1}+\left\|\nabla^{m} \tilde{u}\right\|^{p-1}\right)\left((-\Delta)^{2 m} \tilde{u},(-\Delta)^{k} \sigma_{t}\right) \\
= & M^{\prime \prime}(\xi)(1-a)(\tilde{s}-s)\left(\nabla^{m} \bar{u},\left\|\nabla^{m} u\right\|^{p-1}+\left\|\nabla^{m} \tilde{u}\right\|^{p-1}\right)\left((-\Delta)^{2 m} \tilde{u},(-\Delta)^{k} \sigma_{t}\right) \\
\leq & C_{8}\left\|\nabla^{2 m+k} \bar{u}\right\|^{2}\left\|\nabla_{2 m+k} \sigma_{t}\right\| .
\end{aligned}
$$$$
I_{2}=M^{\prime}(s)\left(\nabla^{m} \bar{u},\left\|\nabla^{m} u\right\|^{p-1}+\left\|\nabla^{m} \tilde{u}\right\|^{p-1}\right)\left((-\Delta)^{2 m} \tilde{u},(-\Delta)^{k} \sigma_{t}\right)
$$$$
-p M^{\prime}(s)\left(\nabla^{m} \bar{u}+\nabla^{m} \sigma,\left\|\nabla^{m} u\right\|^{p-1}\right)\left((-\Delta)^{2 m} \tilde{u},(-\Delta)^{k} \sigma_{t}\right)
$$$$
=M^{\prime}(s)\left(\nabla^{m} \bar{u},\left\|\nabla^{m} \tilde{u}\right\|^{p-1}\right)\left((-\Delta)^{2 m} \tilde{u},(-\Delta)^{k} \sigma_{t}\right)
$$$$
-p M^{\prime}(s)\left(\nabla^{m} \sigma,\left\|\nabla^{m} u\right\|^{p-1}\right)\left((-\Delta)^{2 m} \tilde{u},(-\Delta)^{k} \sigma_{t}\right)
$$ 


$$
\begin{gathered}
\leq M^{\prime}(s)\left\|\nabla^{2 m+k} \bar{u}\right\|^{2}\left\|\nabla^{m} \tilde{u}\right\|^{p-1}\left\|\nabla^{2 m} \tilde{u}\right\|\left\|\nabla^{2 m+k} \sigma_{t}\right\| \\
+p M^{\prime}(s)\left\|\nabla^{m+k} \sigma\right\|\left\|\nabla^{m} u\right\|^{p-1}\left\|\nabla^{2 m} \tilde{u}\right\|\left\|\nabla^{2 m+k} \sigma_{t}\right\| \\
\leq C_{9}\left(\left\|\nabla^{2 m+k} \bar{u}\right\|^{2}+\left\|\nabla^{2 m+k} \sigma\right\|\right)\left\|\nabla^{2 m+k} \sigma_{t}\right\| . \\
I_{3}=-p M^{\prime}(s)\left\|\nabla^{m} u\right\|^{p-2}\left\|\nabla^{m} u\right\|\left\|\nabla^{m} U\right\|\left((-\Delta)^{2 m} \tilde{u},(-\Delta)^{k} \sigma_{t}\right) \\
+p M^{\prime}(s)\left\|\nabla^{m} u\right\|^{p-2}\left\|\nabla^{m} u\right\|\left\|\nabla^{m} U\right\|\left((-\Delta)^{2 m} u,(-\Delta)^{k} \sigma_{t}\right) \\
=p M^{\prime}(s)\left\|\nabla^{m} u\right\|^{p-2}\left(\nabla^{m} \tilde{u}-\nabla^{m} u-\nabla^{m} \sigma, \nabla^{m} u\right)\left((-\Delta)^{2 m} \bar{u},(-\Delta)^{k} \sigma_{t}\right) \\
\leq p M^{\prime}(s)\left\|\nabla^{2 m+k} \bar{u}\right\|^{2}\left\|\nabla^{m} u\right\|^{p-2}\left\|\nabla^{2 m+k} \sigma_{t}\right\| \\
+\left\|\nabla^{m+k} \sigma\right\| \nabla^{m} u\left\|^{p-1}\right\| \nabla^{2 m} \bar{u}\|\| \nabla^{2 m+k} \sigma_{t} \| \\
\leq C_{10}\left(\left\|\nabla^{2 m+k} \bar{u}\right\|^{2}+\left\|\nabla^{2 m+k} \sigma\right\|\right)\left\|\nabla^{2 m+k} \sigma_{t}\right\| .
\end{gathered}
$$

It can be obtained from hypothesis (H1), Holder's inequality, Young's inequality, Poincare's inequality and differential mean value theorem, let $w_{t}=u_{t}-\overline{u_{t}}$

$$
\begin{aligned}
& \left(g\left(u_{t}\right)-g\left(\overline{u_{t}}\right)+g^{\prime}\left(u_{t}\right) U_{t},(-\Delta)^{k} \sigma_{t}\right) \\
& =\left(g^{\prime}(x) w_{t},(-\Delta)^{k} \sigma_{t}\right)-\left(g^{\prime}\left(u_{t}\right) w_{t},(-\Delta)^{k} \sigma_{t}\right)-\left(g^{\prime}\left(u_{t}\right) \sigma,(-\Delta)^{k} \sigma_{t}\right) \\
& \leq(\theta-1)\left(g^{\prime \prime}(\xi)\left\|w_{t}\right\|^{2},(-\Delta)^{k} \sigma_{t}\right)-\left(g^{\prime}\left(u_{t}\right) \sigma,(-\Delta)^{k} \sigma_{t}\right) \\
& \leq C_{11}\left(\nabla^{2 m+k}\left\|w_{t}\right\|^{2}+\nabla^{k}\left\|\sigma_{t}\right\|\right) \nabla^{2 m+k}\left\|\sigma_{t}\right\| .
\end{aligned}
$$

Substitute (4.9)-(4.13) into Formula (4.8), then it can be obtained by Young's inequality and Poincare's inequality

$$
\begin{aligned}
& \frac{\mathrm{d}}{\mathrm{d} t}\left(\left\|\mu D^{2 m+k} \sigma\right\|^{2}+\left\|D^{k} \sigma_{t}\right\|^{2}\right) \\
& \leq C_{12}\left(\left\|\mu D^{2 m+k} \sigma\right\|^{2}+\left\|D^{k} \sigma_{t}\right\|^{2}\right)+C_{13}\left(\left\|\mu D^{2 m+k} \sigma\right\|^{4}\right)+C_{14}\left(\left\|\nabla^{2 m+k} w_{t}\right\|^{2}\right) .
\end{aligned}
$$

According to Gronwall's inequality

$$
\left\|D^{k} \sigma_{t}\right\|^{2}+\mu\left\|D^{2 m+k} \sigma_{t}\right\|^{2} \leq C_{15} \cdot \mathrm{e}^{C_{12} t}\left\|\left(\xi_{1}, \xi_{2}\right)\right\|_{E_{k}}^{4} .
$$

When $\left\|\left(\xi_{1}, \xi_{2}\right)\right\|_{E_{k}}^{2} \rightarrow 0$, there is

$$
\frac{\|\overline{\phi(t)}-\phi(t)-U(t)\|}{\left\|\left(\xi_{1}, \xi_{2}\right)\right\|_{E_{k}}^{2}} \leq C_{15} \mathrm{e}^{C_{12} t}\left\|\left(\xi_{1}, \xi_{2}\right)\right\|_{E_{k}}^{2} \rightarrow 0 .
$$

The theorem is proved.

Theorem 4.2. Under the condition of theorem 3.3, the family of global attractor $A_{k}$ of Problems (1.1)-(1.3) have finite Hansdorff dimension and Fractal dimension, and $d_{H}\left(A_{k}\right)<\frac{2 n}{5}, d_{F}\left(A_{k}\right)<\frac{7 n}{5}$.

Proof: assume $\Psi=R_{\xi} \Phi=(u, v)^{\mathrm{T}}, \Phi=\left(u, v_{t}\right), v=u_{t}+\varepsilon u$, then $R_{\varepsilon}:\left(u, u_{t}\right)^{\mathrm{T}} \rightarrow\left(u, u_{t}+\varepsilon u\right)$ is an isomorphic mapping. 
Linearize Equation (4.1)

$$
\begin{aligned}
& \Psi_{t}+\Lambda_{\varepsilon} \Psi+\bar{g}(\Psi)=\bar{f} . \\
& \Psi(0)=\left(u_{0}, u_{1}+\varepsilon u_{0}\right)^{\mathrm{T}} .
\end{aligned}
$$

where $\Psi=\left\{u, u_{t}+\varepsilon u\right\}^{\mathrm{T}}, \bar{g}(\Psi)=\left\{0, g\left(u_{t}\right)\right\}^{\mathrm{T}}, \bar{f}=\{0, f(x)\}^{\mathrm{T}}$

$$
\begin{gathered}
\Lambda_{\varepsilon}=\Lambda(\Psi)=\left(M\left(\| A^{\left.\frac{m}{2} u \|_{p}^{p}-\varepsilon \beta\right) A^{2 m}+\varepsilon^{2} I} \quad \beta A^{2 m}-\varepsilon I\right)\right. \\
\Psi_{t}:=F(\Psi)=\bar{f}-\Lambda_{\varepsilon} \Psi-\bar{g}(\Psi) . \\
P_{t}=F_{t}(\Psi) P . \\
P_{t}+\Lambda_{\varepsilon} P+\bar{h}(\Psi)=0 .
\end{gathered}
$$

where $P=\left\{U, U_{t}+\varepsilon U\right\}^{\mathrm{T}}, \overline{g_{t}}(\Psi) P=\left\{0, g_{t}\left(u_{t}\right) U\right\}^{\mathrm{T}}$. $U$ is the solution of (4.16).

For a fixed $\left(u_{0}, v_{0}\right) \in E_{k}$, Let $\xi_{1}, \xi_{2}, \cdots, \xi_{n}$ be $\mathrm{n}$ elements in $E_{k}$. letting $U_{1}(t), U_{2}(t), \cdots, U_{n}(t)$ is several solutions of linear Equation (4.1) with initial value of $U_{1}(0)=\xi_{1}, U_{2}(0)=\xi_{2}, \cdots, U_{n}(0)=\xi_{n}$. Available by direct calculation

$$
\begin{aligned}
& \left\|U_{1}(t) \Lambda U_{2}(t) \Lambda \cdots \Lambda U_{n}(t)\right\|_{\Lambda E_{k}}^{2} \\
& =\left\|\xi_{1} \Lambda \xi_{2} \Lambda \cdots \Lambda \xi_{n}\right\|_{\Lambda E_{k}}^{2} \exp \left(-\int_{0}^{t} t r F_{t}^{\prime}(\Psi) Q_{n}(\tau) \mathrm{d} \tau\right) .
\end{aligned}
$$

where $\Lambda$ represents the outer product, tr stands for trace, $Q_{n}(\tau)$ represents the orthogonal projection from $E_{k}$ to the subspace generated by $U_{1}(t), U_{2}(t), \cdots, U_{n}(t)$.

For a given moment $\tau$, let $w_{j}(\tau)=\left(\xi_{j}(\tau), \eta_{j}(\tau)\right)^{\mathrm{T}}, j=1,2, \cdots, n$ be the standard orthogonal basis of space $U_{1}(t), U_{2}(t), \cdots, U_{n}(t)$.

Define the inner product over $E_{k}$

$$
((\xi, \eta),(\bar{\xi}, \bar{\eta}))=\left(D^{2 m+k} \xi, D^{2 m+k} \bar{\xi}\right)+\left(D^{k} \eta, D^{k} \bar{\eta}\right) .
$$

To sum up, there are

$$
\operatorname{tr} F_{t}(\Psi(\tau)) Q(\tau)=\sum_{j=1}^{n}\left(F_{t}(\Psi(\tau)) Q_{n}(\tau) w_{j}(\tau), w_{j}(\tau)\right)_{E_{k}} .
$$

where

$$
\left(F_{t}(\Psi(\tau)) w_{j}(\tau), w_{j}(\tau)\right)_{E_{k}}=-\left(\Lambda_{\varepsilon} w_{j}, w_{j}\right)_{E_{k}}-\left(g_{t}\left(u_{t}\right) w_{j}, w_{j}\right) .
$$

$$
\begin{aligned}
& \left(\Lambda_{\varepsilon} \omega_{j}, \omega_{j}\right)_{E_{k}} \\
& =\left(\left(\varepsilon \xi_{j}-\eta_{j},\left(M\left\|D^{m} u\right\|_{p}^{p}-\beta \varepsilon\right) A^{2 m} \xi_{j}+\varepsilon^{2} \xi_{j}+D \xi_{j}+\beta A^{2 m} \eta_{j}-\varepsilon \eta_{j}\right),\left(\xi_{j}, \eta_{j}\right)\right) \\
& =\varepsilon\left\|D^{2 m+k} \xi_{j}\right\|^{2}+\left(M\left\|D^{m} u\right\|_{p}^{p}-\beta \varepsilon-1\right)\left(D^{2 m+k} \xi_{j}, D^{2 m+k} \eta_{j}\right) \\
& +\varepsilon^{2}\left(D^{k} \xi_{j}, D^{k} \eta_{j}\right)+\left(D^{k} D \xi_{j}, D^{k} \eta_{j}\right)+\beta\left\|D^{2 m+k} \eta_{j}\right\|^{2}-\varepsilon\left\|D^{k} \eta_{j}\right\|^{2}
\end{aligned}
$$




$$
\begin{aligned}
& \geq \varepsilon\left\|D^{2 m+k} \xi_{j}\right\|^{2}-(M-\beta \varepsilon-1)\left\|D^{2 m+k} \xi_{j}\right\|\left\|D^{2 m+k} \eta_{j}\right\|+\beta\left\|D^{2 m+k} \eta_{j}\right\|^{2} \\
& -\varepsilon\left\|D^{k} \eta_{j}\right\|^{2}-\frac{\varepsilon^{2}}{\lambda_{j}^{m}}\left\|D^{2 m+k} \xi_{j}\right\|\left\|D^{k} \eta_{j}\right\| \\
& -p M^{\prime}\left\|D^{m} u\right\|_{p}^{p}\left\|D^{m} u\right\|_{L^{2 p-2}(\Omega)}^{p-1}\left\|D^{m} \xi_{j}\right\|\left\|D^{2 m+k} u\right\|\left\|D^{2 m+k} \eta_{j}\right\| \\
& \geq\left(\varepsilon-\frac{\varepsilon}{2}\right)\left\|D^{2 m+k} \xi_{j}\right\|^{2}+\left(\frac{\beta}{2}-\frac{1}{2 \varepsilon}\right)\left\|D^{2 m+k} \eta_{j}\right\|^{2}-\varepsilon\left\|D^{k} \eta_{j}\right\|^{2} \\
& -\frac{\varepsilon^{2}}{2 \lambda_{j}^{m}}\left(\left\|D^{2 m+k} \xi_{j}\right\|^{2}+\left\|D^{k} \eta_{j}\right\|^{2}\right)-\frac{C_{16}}{\beta}\left\|D^{m} \xi_{j}\right\|^{2} \\
& \geq\left(\frac{\varepsilon}{2}-\frac{\varepsilon^{2}}{2 \lambda_{j}^{m}}-\frac{C_{16}}{\beta \lambda_{j}^{\frac{m+k}{2}}}\right)\left\|D^{2 m+k} \xi_{j}\right\|^{2}+\left(\left(\frac{\beta}{2}-\frac{1}{2 \varepsilon}\right) \lambda_{1}^{m}-\varepsilon-\frac{\varepsilon^{2}}{2 \lambda_{j}^{m}}\right)\left\|D^{k} \eta_{j}\right\|^{2} \\
& -\frac{C_{16}}{\beta}\left\|D^{m} \xi_{j}\right\|^{2} \\
& a=\min \left\{\frac{\varepsilon}{2}-\frac{\varepsilon^{2}}{2 \lambda_{j}^{m}}-\frac{C_{16}}{\beta \lambda_{j}^{\frac{m+k}{2}}},\left(\frac{\beta}{2}-\frac{1}{2 \varepsilon}\right) \lambda_{1}^{m}-\varepsilon-\frac{\varepsilon^{2}}{2 \lambda_{j}^{m}}\right\} .
\end{aligned}
$$

Present hypothesis $\left\{u_{0}, u_{1}\right\} \in A$, according to theorem 3.3, $A$ is a bounded absorption set in $E_{1}$.

$\psi(t)=\left\{u(t), u_{t}(t)+\varepsilon u(t)\right\} \in D(A) ; D(A)=\{u \in v, A u \in H\}$, there is $s \in[0,1]$ with mapping $h_{1}: D(A) \rightarrow p\left(v_{s}, H\right)$

$$
\begin{gathered}
R_{A}=\sup _{(w, \eta) \in A}|A \xi|<\infty, \\
\sup _{u \in D(A),\left|A_{u}<R_{A}\right|}\left|g_{t}\left(u_{t}\right)\right|_{p\left(v_{s}, H\right)} \leq R<\infty,
\end{gathered}
$$

where $\left\|g_{t}\left(u_{t}\right) \omega_{j}, \eta_{j}\right\|$ satisfy

$$
\left\|g_{t}\left(u_{t}\right) \omega_{j}, \eta_{j}\right\| \leq r\left\|\omega_{j}\right\|\left\|_{s}\right\| \eta_{j} \| .
$$

Comprehensive the above contents are as follows

$$
\begin{aligned}
\left(F_{t}(\psi) \omega_{j}, \omega_{j}\right)_{E_{k}} & \leq-a\left(\left\|D^{2 m+k} \xi_{j}\right\|^{2}+\left\|D^{k} \eta_{j}\right\|^{2}\right)-\frac{C_{14}}{\beta}\left\|D^{m} \xi_{j}\right\|^{2}+r\left\|\omega_{j}\right\|\left\|_{s}\right\| \eta_{j} \| \\
& \leq-\frac{a}{2}\left(\left\|D^{2 m+k} \xi_{j}\right\|^{2}+\left\|D^{k} \eta_{j}\right\|^{2}\right)+\frac{r}{2}\left\|\xi_{j}\right\|^{2} .
\end{aligned}
$$

where

$$
\begin{aligned}
\left\|D^{2 m+k} \xi_{j}\right\|^{2}+\left\|D^{k} \eta_{j}\right\|^{2} & =1 . \\
\sum_{j=1}^{n}\left(F_{t}(\psi(\tau)) \omega_{j}(\tau), \omega_{j}(\tau)\right)_{E_{k}} & \leq-\frac{n a}{2}+\frac{r}{2}\left\|\xi_{j}\right\|^{2} .
\end{aligned}
$$

Almost any thas

$$
\sum_{j=1}^{n}\left\|\xi_{j}\right\|_{s}^{2} \leq \sum_{j=1}^{n-1} \lambda_{j}^{s-1}
$$


So

$$
T_{r} F_{t}\left(\psi(\tau) Q_{n}(\tau)\right) \leq-\frac{n a}{2}+\frac{r}{2} \sum_{j=1}^{n-1} \lambda_{j}^{s-1}
$$

Set

$$
\begin{gathered}
q_{n}(t)=\sup _{\psi_{0} \in A_{D_{j}} \in E_{K}, D^{K} \eta_{j} \mid \leq 1} \sup _{t}\left(\frac{1}{t} \int_{0}^{t} \operatorname{tr} F_{t}\left(s(\tau) \psi_{0}\right) Q_{n}(\tau)\right) \mathrm{d} \tau . \\
q_{N}=\lim _{t \rightarrow \infty} \sup q_{N} .
\end{gathered}
$$

It can be known from (4.32)

$$
q_{n} \leq-\frac{n a}{2}+\frac{r}{2} \sum_{j=1}^{n-1} \lambda_{j}^{s-1}
$$

Therefore, the Lyapunov exponent $\mu_{j}(j \in N)$ of $B_{0 k}$ is uniformly bounded

$$
\mu_{1}+\mu_{2}+\cdots+\mu_{n} \leq-\frac{n a}{2}+\frac{r}{2} \sum_{j=1}^{n-1} \lambda_{j}^{s-1}
$$

From the above knowledge, there are $n>1$ and $s \in[0,1]$,

$$
\begin{aligned}
\left(q_{j}\right)_{+} & \leq-\frac{n a}{2}+\frac{r}{2} \sum_{j=1}^{n-1} \lambda_{j}^{s-1} \leq \frac{r}{2} \sum_{j=1}^{n-1} \lambda_{j}^{s-1} \leq \frac{n a}{7} . \\
q_{n} & \leq-\frac{n a}{2}\left(1-\frac{r}{n a} \sum_{j=1}^{n-1} \lambda_{j}^{s-1}\right) \leq-\frac{5 n a}{14} .
\end{aligned}
$$

So

$$
\max _{1 \leq j \leq n} \frac{\left(q_{j}\right)_{+}}{\left|q_{n}\right|} \leq \frac{2}{5} .
$$

Thus, we can get the conclusion

$$
d_{H}\left(A_{k}\right)<\frac{2 n}{5}, d_{F}\left(A_{k}\right)<\frac{7 n}{5} .
$$

\section{Conflicts of Interest}

The authors declare no conflicts of interest regarding the publication of this paper.

\section{References}

[1] Nakao, M. and Yang, Z.J. (2007) Gobal Attractor for Some Quasi-Linear Wave Equations with a Strong Dissipation. Advances in Mathematical Sciences and Applications, 17, 89-105.

[2] Fan, X.M. and Zhou, S.F. (2004) Kernel Sections for Non-Autonomous Strongly Damped Wave Equation of Non-Degnerate Kirchhoff-Type. Applied Mathematics and Computation, 158, 253-266. https://doi.org/10.1016/j.amc.2003.08.147

[3] Messaoudi, S.A. and Houari, B.Said. (2007) A Blow-Up Result for Ahigher-Order Nonlinear Kirchhoff-Type Hyperbolic Equation. Appiled Mathematics Letters, 20, 866-871. https://doi.org/10.1016/j.aml.2006.08.018

[4] Li, F.C. (2004) Global Existence and Blow-Up of Solutions for a Higher-Order Kir- 
chhoff-Type Equation with Nonlinear Dissipation. Applied Mathematics Letters, 17, 1409-1414. https://doi.org/10.1016/j.am1.2003.07.014

[5] Yang, Z.J. and Jin, B.X. (2009) Global Attractor for a Class of Kirchhoff Models. Journal of Mathematical Physics, 50, Article ID: 032701. https://doi.org/10.1063/1.3085951

[6] Cheng, J.L. and Yang, Z.J. (2011) Asymptotic Behavior of the Kirchhoff Type Equation. Acta Mathematica Scientia, 31, 1008-1021.

[7] Lin, G.G. and Wang, W. (2017) The Global Attractors for the Higher-Order Nonlinear Kirchhoff-Type Equation with Nonlinear Damped Terms. European Journal of Mathematics and Computer Science, 4, 25-36.

[8] Lin, G.G. and Zhu, C.Q. (2019) Asymptotic State of Solutions for a Class of Nonlinear Higher Order Kirchhoff Type Equations. Journal of Yunnan University: Natural Sciences Edition, 41, 867-875.

[9] Lin, G.G. (2019) Dynamic Properties of Several Kinds of the K Equations. Chongqing University Press, Chongqing.

[10] Yang, Z.J., Ding, P.Y. and Li, L. (2016) Longtime Dynamics of the Kirchhoff Equation with Fractional Damping and Supercritical Nonlinearity. Journal of Mathematical Analysis and Applications, 442, 485-510. https://doi.org/10.1016/j.jmaa.2016.04.079

[11] Lin, G.G. and Guan, L.P. (2019) The Family of Global Attractor and Their Dimension Estimates for Strongly Damped High-Order Kirchhoff Equation. Acta Analysis Functionalis Applicata, 21, 268-281.

[12] Lin, G.G. and Yang, S.M. (2017) Hausdorff Dimension and Fractal Dimension of the Global Attractor for the Higher-Order Coupled Kirchhoff-Type Equations. Journal of Applied Mathematics and Physics, 5, 2411-2424. https://doi.org/10.4236/jamp.2017.512197

[13] Gao, Y.L., Sun, Y.T. and Lin, G.G. (2016) The Global Attractors and Their Hausdorff and Fractal Dimensions Estimation for the Higher Order Nonlinear Kirchhoff-Type Equation with Strong Linear Damping. International Journal of Modern Nonlinear Theory and Application, 5, 185-202. https://doi.org/10.4236/ijmnta.2016.54018

[14] Lin, G.G. and Chen, Y.H. (2020) A Family of Global Attractors for the Higher-Order Kirchhoff-Type Equations and Its Dimension Estimation. American Journal of Applied Mathematics, 8.

[15] Yang, Z.J. and Wang, Y.Q. (2010) Global Attractor for the Kirchhoff Type Equations with a Strong Dissipation. Journal of Differential Equations, 249, 3258-3278. https://doi.org/10.1016/j.jde.2010.09.024 